\title{
Preditores de mortalidade hospitalar no paciente idoso portador de doença arterial coronária
}

\author{
José Carlos R. IGLÉZIAS*, José Lima OLIVEIRA Jr. *, Luís Alberto O. DALLAN*, \\ Artur LOURENÇÃO Jr.*, Noedir A. G. STOLF*
}

RBCCV 44205-535

\begin{abstract}
Iglézias J C R, Oliveira Jr. J L, Dallan L A O, Lourenção Jr. A, Stolf N A G - Preditores de mortalidade hospitalar no paciente idoso portador de doença arterial coronária. Rev Bras Cir Cardiovasc 2001; 16(2): 94-104.

RESUMO: Introdução: O receio em nosso meio, em se retardar, erroneamente, a revascularização do miocárdio ( $\mathrm{RM}$ ) em pacientes idosos determinou a realização deste estudo.

Casuística e Métodos: Um total de 361 pacientes foram, consecutivamente, submetidos a RM entre 1992 e 1995, dos quais 30,7\% eram mulheres, 69,3\% homens; 36,7\% encontravam-se em classe funcional III/IV. Foi realizada análise univariada com 19 fatores pré-operatórios e, a seguir, multivariada (regressão logística) com as variáveis que mostraram associação significativa $(p<0,005)$.

Resultados: Os fatores prognósticos da morbidade operatória foram: diabete melito, ICC, angina instável. Os pós-operatórios foram: acidente vascular cerebral, insuficiência renal, infecção e suporte respiratório prolongado.

Conclusão: A RM pode ser realizada em pacientes com idade avançada, acompanhada de excelentes resultados (baixa mortalidade operatória), especialmente pacientes em classe funcional de ICC não muito avançada, propiciando melhora significativa da qualidade de vida.
\end{abstract}

DESCRITORES: Coronariopatia, cirurgia. Revascularização miocárdica, mortalidade. Mortalidade hospitalar. Revascularização miocárdica, idoso.

\section{INTRODUÇÃO}

A doença aterosclerótica das artérias coronárias epicárdicas (DAC), causa mais comum da isquemia miocárdica, ao reduzir a luz arterial, provoca diminuição do fluxo sangüíneo coronário, restringindo a perfusão miocárdica já em estado basal ou limitando aumentos proporcionais quando há necessidade de maior fluxo. Menos freqüentemente, trombos arteriais, espasmos e êmbolos coronários podem determinar redução do fluxo sangüíneo nas artérias coronárias epicárdicas, levando a um desequilíbrio entre a oferta e a demanda de $\mathrm{O}_{2}$ a nível miocárdico(1). Com o aumento da expectativa de vida da população, o número de idosos vem crescendo em termos relativo e absoluto. Estudos realizados nos Estados Unidos ${ }^{(2-5)}$, no início da década de 80 , mostraram que $11 \%$ da população era constituída por idosos, estimativas realizadas a partir destes dados mostram que $40 \%$ dos norte-

Trabalho realizado na Divisão Cirúrgica do Instituto do Coração do Hospital das Clínicas da Faculdade de Medicina da Universidade de São Paulo. São Paulo, SP, Brasil.

Apresentado ao 27ํㅡㄹ Congresso Nacional de Cirurgia Cardíaca. Rio de Janeiro, RJ, 23 a 25 de março de 2000.

* Do Instituto do Coração do Hospital das Clínicas da Faculdade de Medicina da Universidade de São Paulo.

Endereço para correspondência: José Carlos Rossini Iglézias. Av. Dr. Enéas de Carvalho Aguiar, 44. Divisão Cirúrgica. São Paulo, SP, Brasil. CEP: 05403-000. Tel. (11) 3021-1870. e.mail: dcirossini@incor.usp.br 
Iglézias J C R, Oliveira Jr. J L, Dallan L A O, Lourenção Jr. A, Stolf N A G - Preditores de mortalidade hospitalar no paciente idoso portador de doença arterial coronária. Rev Bras Cir Cardiovasc 2001; 16(2): 94-104.

americanos atingirão 80 anos de idade e que os idosos irão compor cerca de $20 \%-25 \%$ de sua população, na primeira metade do próximo sécuIo ${ }^{(1)}$. Esta tendência é verificada no Brasil, onde a população de idosos já atinge cerca de 7 milhões, devendo ter, segundo o IBGE (Fundação IBGE Anuário Estatístico do Brasil. Rio de Janeiro, 1981, 1985 , 1986), um aumento progressivo da atual velocidade de crescimento $(3,5 \%$ ao ano) nos próximos anos, o que, por si só, tem repercussões socioeconômicas extremamente importantes. Como a prevalência da DAC aumenta com a idade, é de se esperar que a demanda por Serviços Cardiológicos aumente nos próximos anos. Tem-se verificado, nas últimas décadas, uma redução progressiva da mortalidade atribuível à DAC, embora a incidência absoluta da doença continue a aumen$\operatorname{tar}(2,3,6)$. Diversos modelos de estudo epidemiológico prevêem que, com as atuais taxas de crescimento da população idosa, ter-se-á, não só um aumento na incidência da DAC, mas também da mortalidade total e do custo econômico em cerca de $50 \%$ por volta do ano 2010 , devendo-se ter, provavelmente, nas próximas décadas, um aumento progressivo do número de pacientes idosos que se apresentam aos Serviços de Cirurgia Cardíaca (3). Quando o tratamento clínico é insuficiente para a melhora sintomática, a revascularização do miocárdio (RM) deve ser considerada. Entretanto, um procedimento de grande porte como este está associado à elevada morbidade e mortalidade operatórias, sendo bem mais dispendioso do que a terapêutica medicamentosa. A introdução da operação de RM, há mais de três décadas, possibilitou nova e eficaz terapêutica a pacientes com doença aterosclerótica avançada, com alívio sintomático em grande número de pacientes e aumento da sobrevida em alguns subgrupos. As operações de RM têm-se tornado cada vez mais freqüentes em pacientes com idade avançada, que apresentam um acometimento coronário mais extenso, com artérias tortuosas, rígidas, calcificadas, além de outros fatores que podem contribuir negativamente para os resultados da RM nessa faixa etária. Em pacientes com idade avançada a indicação da RM deve se basear num balanço cuidadoso entre os riscos e potenciais benefícios, o que requer uma avaliação cuidadosa do quadro clínico, da anatomia das artérias coronárias e da função ventricular, uma vez que estes fatores poderão determinar o sucesso imediato do procedimento. Para muitos pacientes idosos um estilo de vida ativo e independente é mais importante que a longevidade em si. Às portas do século XXI, a DAC continua sendo a principal causa de óbito nos países desenvolvidos e uma das principais em nosso meio, justificando-se o grande número de estudos, que vem sendo realizado a cada ano (1). A fim de verificar os fatores correlacionados com a morbidade e mortalidade operatória da RM em pacientes com idade avançada, levantamos os dados referentes a 361 pacientes consecutivos, com mais de 70 anos de idade, submetidos, isoladamente, à primeira operação de RM no Instituto do Coração da Faculdade de Medicina da Universidade São Paulo (InCor FMUSP), entre 1992 e 1995.

\section{CASUÍSTICA E MÉTODOS}

Este estudo populacional retrospectivo abrange todos os pacientes, consecutivos, com idade igual ou superior a 70 anos, submetidos a sua primeira operação de RM. As variáveis incluídas neste estudo são descritas na Tabela 1. Os dados foram coletados diretamente a partir dos prontuários dos pacientes, ou por contato telefônico nos casos em que faltavam informações. A fração de ejeção (FE) era considerada má quando menor que $50 \%$ e, boa se superior a $50 \%$. A classificação do grau de insuficiência cardíaca/paciente seguiu os critérios estabelecidos pela New York Heart Association (NYHA).

TABELA 1

VARIÁVEIS INCLUÍDAS NESTE ESTUDO

\section{PRÉ-OPERATÓRIAS}

Idade
Sexo
Diabete melito
Hipertensão arterial sistêmica
Tabagismo
Dislipidemia
Obesidade
Infarto agudo do miocárdio
Classificação de angina pectoris
Classificação funcional NYHA
Pressão diastólica final de ventrículo esquerdo
№ de ramos arteriais acometidos
Acometimento de tronco de artéria coronária esquerda
Fração de ejeção
Urgência da operação

\section{PÓS-OPERATÓRIAS}

Baixo débito cardíaco

Acidente vascular cerebral

Insuficiência respiratória

Infecção

Suporte ventilatório prolongado

Óbito 
Iglézias J C R, Oliveira Jr. J L, Dallan L A O, Lourenção Jr. A, Stolf N A G - Preditores de mortalidade hospitalar no paciente idoso portador de doença arterial coronária. Rev Bras Cir Cardiovasc 2001; 16(2): 94-104.

Foram considerados portadores de angina instável todos os pacientes que apresentavam dor há menos de 3 meses, progressiva ou em repouso. O número de ramos arteriais acometidos, a pressão diastólica final do ventrículo esquerdo $\left(\mathrm{PD}_{2}\right)$ e o acometimento do tronco da artéria coronária esquerda (TCE) era determinado a partir da análise do estudo contrastado das artérias coronárias e da função ventricular. A presença de diabete melito, hipertensão arterial sistêmica (HAS), tabagismo e dislipidemia era verificada diretamente a partir das informações colhidas nos prontuários, estabelecendo-se contato telefônico quando necessário. Foram considerados obesos os pacientes com IMC>25. Considerou-se como infarto agudo do miocárdio (IAM) prévio, o ocorrido até, pelo menos, duas semanas antes da operação. As operações eram consideradas eletivas quando os pacientes eram internados eletivamente e a operação realizada nos dias subseqüentes e, não eletiva, quando os doentes eram internados após IAM, em choque cardiogênico ou edema agudo de pulmão, necessitando de combinação de suporte inotrópico, balão intraaórtico (BIA) e ventilação mecânica. O número de enxertos realizados era definido pelo número de anastomoses distais. A presença de baixo débito foi considerada em todos os pacientes com instabilidade hemodinâmica, havendo necessidade de drogas vasoativas e nos casos mais graves, BIA no pós-operatório imediato. Os pacientes com nível sérico de creatinina superior a 2 $\mathrm{mg} / \mathrm{dl}$, no pós-operatório foram considerados portadores de insuficiência renal (IR). A ocorrência de infecção era verificada pelo quadro clínico, exames laboratoriais e necessidade de antibioticoterapia prolongada, AVC pelas alterações neurológicas localizatórias ou do nível de consciência persistentes por mais de 24 horas. Todos os óbitos ocorridos durante a internação ou no $1^{\circ}$ mês após a operação, foram computados como mortalidade operatória. Quanto à análise estatística, inicialmente todas as variáveis foram analisadas descritivamente em cada um dos grupos através da observação dos valores mínimos e máximos, e do cálculo de médias e desvios-padrão. Para a identificação dos fatores de risco para a morbidade e mortalidade operatória foi utilizada a técnica da estatística de regressão logística, que consiste no ajuste de um modelo de regressão cuja resposta é a probabilidade de ocorrência de alguma complicação ou de óbito na RM em pacientes com idade avançada. Esta técnica consiste, inicialmente, na avaliação da associação univariada de cada variável com a variável resposta (complicação pós-operatória ou óbito). Essa avaliação foi feita através dos testes T de Student e Qui-quadrado (Software Ep.
Info 6.0) considerando-se como nível de significância $5 \%$. Após esta pré-seleção, ajustou-se o modelo de regressão logística multivariado com processo de seleção Stepwise para a obtenção do melhor modelo. O nível de significância utilizado para os testes foi de $5 \%$. Os cálculos foram realizados através do sistema SAS (Statistical Analysis System) (6-9). O programa MULTLR Fundação Ludwig - Institute for Cancer Research SP Branch também foi utilizado, na análise da correlação das complicações pós-operatórias com a mortalidade operatória. "Odds ratio" (OR) e teste da razão da máxima verossimilhança também foram utilizados quando possível e necessário. Optamos pela inclusão da variável dislipidemia na análise multivariada da mortalidade operatória, por termos encontrado um valor limítrofe $(p$ $=0,051)$ na análise univariada. Houve perda de alguns dados pré-operatórios, fato esperado, considerando-se a estrutura retrospectiva deste estudo; apenas para fração de ejeção esta perda interferiu nos resultados, impedindo uma análise profunda dos valores resposta obtidos, devido à instabilidade dos OR. As complicações pós-operatórias foram agrupadas como uma única variável resposta, alguma complicação (morbidade operatória), para se viabilizar o emprego dos modelos estatísticos utilizados.

\section{RESULTADOS}

\section{Perfil dos Pacientes}

A idade dos pacientes variou de 70 a 90 anos, com média de 73,92 anos e desvio padrão (d.p.) de 3,32. O IMC médio foi de 26,23 (d.p.=346), sendo 18,34 o valor mínimo e 40,20 o máximo, 14,73 foi a $\mathrm{PD}_{2}$ média dos pacientes, variando de 5,0 a 40,0 (d.p.=6,79). A FE média foi 0,62 (d.p. $=0,13$ ), tendo variado de 0,19 a 0,99 . Quanto à permanência pósoperatória no hospital, verificamos um tempo médio de 11,32 dias, variando de 0 a 72 (d.p.=7,42). Dos 361 pacientes estudados, $111(30,7 \%)$ eram do sexo feminino e 250 (69,3\%) do masculino, 128 (35,5\%) apresentavam diabete melito e HAS estava presente em $238(66,1 \%)$. Cento e trinta $(36 \%)$ pacientes eram tabagistas, $163(45,2 \%)$ tinham apresentado IAM previamente. O estudo contrastado das artérias coronárias mostrou que em 119 (32,2\%) pacientes havia acometimento do TCE e que 274 (75,9\%) apresentavam 3 ou 4 ramos arteriais acometidos. Cento e vinte e um $(36,7 \%)$ pacientes encontravamse em classe funcional III/IV e $63,3 \%$ em I/II pela NYHA. Duzentos e sessenta e três $(72,9 \%)$ pacientes apresentavam angina instável e $98(27,1 \%)$ estável. Quanto à FE, verificamos que em $33(23,7 \%)$ 
Iglézias J C R, Oliveira Jr. J L, Dallan L A O, Lourenção Jr. A, Stolf N A G - Preditores de mortalidade hospitalar no paciente idoso portador de doença arterial coronária. Rev Bras Cir Cardiovasc 2001; 16(2): 94-104.

pacientes ela foi menor que $50 \%$ e que em 106

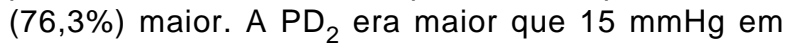
79 (32\%), menor em 168 (68\%). A operação foi eletiva em $311(86,1 \%)$ dos 361 pacientes, não eletiva em 50 (13,9\%). O número médio de enxertos realizados por paciente foi de 2,75 . O perfil préoperatório dos pacientes é descrito nas Tabelas 2 e 3. Verificou-se que, com a operação de revascularização, ocorreu melhora substancial do desempenho cardíaco destes pacientes, o que foi

TABELA 2

DISTRIBUIÇÃO POR FREQÜÊNCIA: SEXO, OBESIDADE, DIABETE MELITO, HIPERTENSÃO ARTERIAL, TABAGISMO, DISLIPIDEMIA, INFARTO PRÉVIO E ACOMETIMENTO DE TCE NOS 361 PACIENTES IDOSOS REVASCULARIZADOS

\begin{tabular}{llll}
\hline VARIÁVEL & CATEGORIA & № & $\%$ \\
\hline Sexo & Masculino & 250 & 69,3 \\
& Feminino & 111 & 30,7 \\
\hline Obesidade & & 201 & 60,5 \\
\hline Diabete melito & 128 & 35,5 \\
\hline Hipertensão & 238 & 66,1 \\
\hline Tabagismo & 130 & 36,0 \\
\hline Dislipidemia & 110 & 30,6 \\
\hline Infarto prévio & 163 & 45,2 \\
\hline TCE acometido & 119 & 33,2 \\
\hline
\end{tabular}

TABELA 3

DISTRIBUIÇÃO POR FREQÜÊNCIA: ANGINA PECTORIS, CLASSE FUNCIONAL DE IC, NÚMERO DE RAMOS ACOMETIDOS, PD, E FE NOS 361 PACIENTES REVASCULARIZADOS

\begin{tabular}{llrc}
\hline VARIÁVEL & CATEGORIA & FREQÜÊNCIA & $\%$ \\
\hline Angina & Estável & 98 & 27,1 \\
& Instável & 263 & 72,9 \\
ICC & I/II & 209 & 63,3 \\
& III/IV & 121 & 36,7 \\
Ramos & 1 ou 2 & 87 & 24,1 \\
acometidos & 3 ou 4 & 274 & 75,9 \\
PD2 & $<=15$ & 168 & 68,0 \\
& $>15$ & 79 & 32,0 \\
FE & $<=50 \%$ & 33 & 23,7 \\
& $>50 \%$ & 106 & 76,3 \\
Operação & Eletiva & 311 & 86,1 \\
& Não eletiva & 50 & 13,9 \\
\hline
\end{tabular}

demonstrado pela melhora da classe funcional de ICC em mais de $90 \%$ dos pacientes pela redução das necessidades de utilização de betabloqueadores e nitratos. A melhoria da qualidade de vida dos pacientes idosos revascularizados fica evidente não só pela redução dos sinais e sintomas de ICC em mais de $90 \%$ dos pacientes, mas também pela redução das queixas de angina, visto que mais de 95\% destes pacientes deixaram o hospital sem referência a dor pré-cordial.

\section{Morbidade Operatória}

As complicações pós-operatórias foram agrupadas em uma única categoria, morbidade operatória, com o intuito de viabilizar a interpretação estatística dos dados, em função das 14 variáveis pré-operatórias analisadas. Cento e setenta e oito $(49,3 \%)$ pacientes apresentavam alguma complicação durante sua evolução pós-operatória. Na Tabela 4 mostramos uma análise diferencial das complicações pósoperatórias apresentadas onde se pode verificar que a intercorrência mais comum no pós-operatório foi baixo débito cardíaco. A infecção pulmonar no pósoperatório respondeu por $53,5 \%$ dos casos (Tabela 5). Dos diversos tipos de infecção, a de maior correlação com óbito foi a mediastinal $(p<0,0001)$.

$\mathrm{Na}$ Tabela 6 estabelecemos uma comparação entre os grupos de pacientes que apresentavam alguma complicação pós-operatória e aqueles que evoluíram sem intercorrências.

Distribuindo morbidade operatória em função das variáveis pré-operatórias, foi maior nos pacientes do sexo feminino $(59,46 \%)$ contra $44,80 \%$ no sexo masculino. Em $75 \%$ dos pacientes diabéticos verificou-se uma ou mais complicações pós-operatórias, enquanto entre os não diabéticos a taxa de

\section{TABELA 4}

TAXA ESCALONADA DE COMPLICAÇÕES PÓSOPERATÓRIAS NOS 361 PACIENTES IDOSOS REVASCULARIZADOS

\begin{tabular}{lrr}
\hline VARIÁVEL & FREQÜÊNCIA & $\%$ \\
\hline Baixo débito cardíaco & 114 & 31,7 \\
\hline Balão intra-aórtico & 25 & 6,9 \\
Acidente vascular cerebral & 33 & 9,1 \\
\hline Insuficiência renal & 53 & 14,7 \\
Infecção & 101 & 28,1 \\
\hline Suporte respiratório prolongado & 81 & 22,4 \\
\hline
\end{tabular}


Iglézias J C R, Oliveira Jr. J L, Dallan L A O, Lourenção Jr. A, Stolf N A G - Preditores de mortalidade hospitalar no paciente idoso portador de doença arterial coronária. Rev Bras Cir Cardiovasc 2001; 16(2): 94-104.

TABELA 5

TIPOS DE INFECCÃO NO PÓS-OPERATÓRIO DE 361 PACIENTES IDOSOS REVASCULARIZADOS

\begin{tabular}{|c|c|c|}
\hline VARIÁVEL & $\%$ & $\%$ ACUMULADA \\
\hline Mediastinal & 4,0 & 4,0 \\
\hline Mediastinal + Outras & 1,0 & 5,0 \\
\hline Outras & 15,8 & 20,8 \\
\hline Pulmonar & 53,5 & 74,3 \\
\hline Pulmonar + Mediastinal & 2,0 & 76,2 \\
\hline Pulmonar + Urinária + Outras & 1,0 & 77,2 \\
\hline Pulmonar + Outras & 2,0 & 79,2 \\
\hline Pulmonar + Urinária & 6,0 & 86,1 \\
\hline Urinária & 12,9 & 99,0 \\
\hline Urinária + Mediastinal & 1,0 & 100,0 \\
\hline
\end{tabular}

complicações foi significativamente menor $(35,19 \%)$. As intercorrências em função do tipo de angina, apresentaram uma taxa 1,5 vezes maior entre os pacientes com angina instável, quando comparados aos portadores de angina estável, razão semelhante à verificada quando se compara a morbidade dos procedimentos não eletivos com os do eletivos. A distribuição da morbidade operatória (taxa de complicações), em função das variáveis analisadas, é descrita nas Tabelas 6 e 7. Quando à análise univariada da morbidade operatória, em função: do sexo, diabete melito, HAS, tabagismo, dislipidemia e IAM prévio, apenas sexo e diabete melito foram estatisticamente significativas, com $p=0,010$ e $p<0,0001$, respectivamente (Tabela 6 ).

Outras variáveis clínicas que apresentaram associação significativa com a morbidade operatória foram: angina instável $(p=0,015)$, ICC $(p<0,0001)$, $\operatorname{PD}_{2}(p=0,007)$ e urgência da operação $(p=0,001)$ - Tabela 7.

Com a análise multivariada procuramos selecionar o conjunto de fatores de maior significância prognóstica da morbidade operatória na RM em pacientes com idade avançada (Tabela 7).

Pelo método de regressão logística e com o Odds rattio verificamos que os fatores de maior prognóstico foram: diabete melito $(p=0,0001)$, ICC $(p=0,0331), P D_{2}(p=0,024)$ e urgência da operação $(p=0,0286)$.

\section{Mortalidade Operatória}

A mortalidade operatória foi de $13(9,1 \%)$ óbitos, taxa semelhante à verificada na literatura
TABELA 6

DISTRIBUIÇÃO DA MORBIDADE OPERATIVA (TAXA DE COMPLICAÇÕES) EM FUNÇÃO: DO SEXO, DIABETE MELITO, HIPERTENSÃO ARTERIAL SISTÊMICA, TABAGISMO, DISLIPIDEMIA, IAM PRÉVIO E OBESIDADE, DE 361 PACIENTES IDOSOS REVASCULARIZADOS

\begin{tabular}{lcrc}
\hline VARIÁVEL & CATEGORIA & \multicolumn{2}{c}{$\begin{array}{c}\text { MORBIDADE } \\
\text { OPERATÓRIA } \\
\text { FREQÜÊNCIA \% }\end{array}$} \\
\hline Sexo & Feminino & 66 & 59,46 \\
& Masculino & 112 & 44,80 \\
Diabete melito & Sim & 96 & 75,00 \\
& Não & 82 & 35,19 \\
Hipertensão & Sim & 118 & 49,58 \\
arterial & Não & 59 & 48,36 \\
\hline Tabagismo & Sim & 63 & 48,46 \\
& Nâo & 115 & 49,76 \\
Dislipidemia & Sim & 57 & 51,82 \\
& Não & 120 & 48,00 \\
IAM prévio & Sim & 89 & 54,60 \\
& Não & 89 & 44,95 \\
\hline Obesidade & Sim & 96 & 47,76 \\
& Não & 61 & 46,56 \\
\hline
\end{tabular}

TABELA 7

DISTRIBUIÇÃO DA MORBIDADE OPERATÓRIA (TAXA DE COMPLICACÕESS), EM FUNÇÃO: ANGINA PECTORIS, CLASSE FUNCIONAL DE ICC, № DE RAMOS ARTERIAIS ACOMETIDOS DE TCE, PD FE E URGÊNCIA DA OPERAÇÃO, DE 361 PACIENTES IDOSOS REVASCULARIZADOS

$\begin{array}{cc}\text { VARIÁVEL CATEGORIA } & \begin{array}{c}\text { MORBIDADE } \\ \text { OPERATÓRIA } \\ \text { FREQÜÊNCIA }(\%)\end{array}\end{array}$

\begin{tabular}{lcrll}
\hline Angina pectoris & Instável & 140 & $(53,23 \%)$ \\
& Estável & 38 & $(38,78 \%)$ \\
\hline NHYA & III/IV & 81 & $(66,94 \%)$ \\
& I-II & 82 & $(39,23 \%)$ \\
\hline \multirow{2}{*}{ № Ramos acometidos } & 3 ou 4 & 143 & $(52,19 \%)$ \\
& 1 ou 2 & 35 & $(40,23 \%)$ \\
\hline \multirow{2}{*}{ Acometimento de TCE } & Sim & 61 & $(51,26 \%)$ \\
& Não & 116 & $(48,54 \%)$ \\
\hline PD & & 80 & $(47,62 \%)$ \\
& $<=15$ & 52 & $(65,82 \%)$ \\
\hline \multirow{2}{*}{ Fração de ejeção } & $>15$ & 23 & $(69,70 \%)$ \\
& $<=50 \%$ & 61 & $(57,55 \%)$ \\
\hline \multirow{2}{*}{ Operação } & $>50 \%$ & 36 & $(72,00 \%)$ \\
& Não eletiva & 142 & $(45,66 \%)$ \\
\hline
\end{tabular}


Iglézias J C R, Oliveira Jr. J L, Dallan L A O, Lourenção Jr. A, Stolf N A G - Preditores de mortalidade hospitalar no paciente idoso portador de doença arterial coronária. Rev Bras Cir Cardiovasc 2001; 16(2): 94-104.

TABELA 8

\begin{tabular}{lcccc}
\hline \multicolumn{5}{c}{ REVISÃO DA LITERATURA SOBRE MORTALIDADE OPERATÓRIA NA REVASCULARIZAÇÃO DO } \\
MIOCÁRDIO EM PACIENTES IDOSOS
\end{tabular}

(Tabela 8). A causa mais comum de óbito foi insuficiência de múltiplos órgãos $(60,60 \%)$, seguida por choque cardiogênico (33,33\%) e AVC (6,07\%). Houve IAM peri-operatório, porém não foi observada relação estatisticamente significativa entre este evento e mortalidade.

Ao estabelecermos uma comparação entre os grupos de pacientes que faleceram ou não, verificamos que a freqüência de mulheres foi maior no grupo de pacientes que faleceram, ao compararmos com o grupo de sobreviventes $(39,39 \%$ versus $29,88 \%)$.

Diabete melito e ICC classe funcional III-IV também foram mais freqüentes no primeiro grupo de pacientes $(63,64 \%$ versus $32,64 \%$ e $66,67 \%$ versus 33,33\%, respectivamente). Quanto à distribuição da mortalidade operatória em função das categorias de variáveis pré-operatórias analisadas (Tabela 9) verificamos que a mortalidade operatória foi maior entre as mulheres $(11,71 \%$ versus $8 \%)$.

Quando comparamos a mortalidade operatória entre os pacientes que apresentam diabete, e os que não, verificamos uma taxa três vezes maior no primeiro grupo.

Quanto à freqüência relativa dos óbitos, em função do tipo de angina apresentada no pré-operatório, verificamos que esta foi seis vezes maior nos portadores de angina instável. Com a análise univariada (Tabelas 9 e 10), pudemos evidenciar a associação significativa do diabete melito $(p<0,0001)$, angina instável $(p=0,004)$, ICC $(p<0,0001), P_{2}(p=0,008)$ e urgência de operação $(p=0,0005)$ com a mortalidade operatória.

Uma análise multivariada e o cálculo do "Odds ratio" com as variáveis clínicas pré-operatórias, triadas previamente com a análise univariada, foram realizados para se identificar o conjunto de fatores de maior significância prognóstica da mortalidade operatória na RM em pacientes com idade avançada (Tabela 11).

Pelo método de regressão logística foram incluídas como variáveis de maior prognóstico da mortalidade operatória: diabete melito $(p=0,0029)$, angina instável $(p=0,0035)$ e ICC $(p=0,00219)$ Tabela 11.

Quanto aos fatores pós-operatórios, os que mostraram associação significativa com o óbito foram AVC $(p=0,0064), I R(p=0,0001)$, infecção $(p=0,0280)$ e suporte ventilatório prolongado $(p=0,0001)$, resultados semelhantes ao da literatura.

\section{COMENTÁRIOS}

Um estudo multicêntrico, retrospectivo, realizado em 1981 por GERSH et al. (10), revelou um 
Iglézias J C R, Oliveira Jr. J L, Dallan L A O, Lourenção Jr. A, Stolf N A G - Preditores de mortalidade hospitalar no paciente idoso portador de doença arterial coronária. Rev Bras Cir Cardiovasc 2001; 16(2): 94-104.

TABELA 9

DISTRIBUICÃO DA MORTALIDADE OPERATÓRIA EM FUNÇÃO: DO SEXO, DIABETE MELITO, HIPERTENSẨO ARTERIAL SISTÊMICA, TABAGISMO, DISLIPIDEMIA, IAM PREVIO E OBESIDADE, DE 361 PACIENTES IDOSOS REVASCULARIZADOS

\begin{tabular}{lcrr}
\hline VARIÁVEL & CATEGORIA & FREQÜÊNCIA & MORTALIDADE \% \\
\hline Sexo & Feminino & 13 & 11,71 \\
& Masculino & 20 & 8,00 \\
Diabete melito & Sim & 21 & 16,41 \\
& Não & 12 & 5,15 \\
Hipertensão arterial & Sim & 23 & 9,66 \\
& Não & 10 & 8,20 \\
Tabagismo & Sim & 15 & 11,54 \\
& Não & 18 & 7,79 \\
Dislipidemia & Sim & 15 & 13,64 \\
& Não & 18 & 7,20 \\
IAM prévio & Sim & 19 & 11,66 \\
\end{tabular}

TABELA 10

DISTRIBUIÇÃO DA MORTALIDADE OPERATÓRIA EM FUNÇÃO: DA ANGINA PECTORIS, CLASSE FUNCIONAL DE ICC, № DE RAMOS ARTERIAIS ACOMETIDOS, ACOMETIMENTO DE TCE, PD, FE E OBESIDADE DE 361 PACIENTES IDOSOS REVASCULARIZADOS

\begin{tabular}{|c|c|c|c|}
\hline VARIÁVEL & CATEGORIA & FREQÜÊNCIA & MORTALIDADE\% \\
\hline Angina pectoris & $\begin{array}{l}\text { Instável } \\
\text { Estável }\end{array}$ & $\begin{array}{r}31 \\
2\end{array}$ & $\begin{array}{r}11,79 \\
2,04\end{array}$ \\
\hline NYHA & $\underset{\text { IIII }}{\text { IIIIV }}$ & $\begin{array}{l}22 \\
11\end{array}$ & $\begin{array}{r}18,18 \\
5,26\end{array}$ \\
\hline № ramos acometidos & $\begin{array}{l}1 \text { ou } 2 \\
3 \text { ou }+\end{array}$ & $\begin{array}{r}6 \\
27\end{array}$ & $\begin{array}{l}6,90 \\
9,85\end{array}$ \\
\hline Acometimento de TCE & $\begin{array}{l}\text { Sim } \\
\text { Não }\end{array}$ & $\begin{array}{l}11 \\
22\end{array}$ & $\begin{array}{l}9,24 \\
9,21\end{array}$ \\
\hline $\mathrm{PD}_{2}$ & $\begin{array}{c}<=15 \\
15\end{array}$ & $\begin{array}{l}10 \\
13\end{array}$ & $\begin{array}{r}5,95 \\
16,46\end{array}$ \\
\hline Fração de ejeção & $\begin{array}{c}<=50 \% \\
>50 \%\end{array}$ & $\begin{array}{l}5 \\
9\end{array}$ & $\begin{array}{r}15,15 \\
8,49\end{array}$ \\
\hline Obesidade & $\begin{array}{l}\text { Sim } \\
\text { Não }\end{array}$ & $\begin{array}{r}22 \\
8\end{array}$ & $\begin{array}{r}10,95 \\
6,11\end{array}$ \\
\hline
\end{tabular}

acréscimo na mortalidade operatória da RM em pacientes com idade avançada. Posteriormente, diversos estudos em pacientes com mais de 70 anos foram realizados com o intuito de reconhecer fatores pré-operatórios possivelmente correlacionados e determinantes desses resultados ${ }^{(5)}$.

Durante a década de 80 , verificou-se uma redução progressiva da mortalidade operatória na RM em pacientes desta faixa etária, que se atribui ao aperfeiçoamento das técnicas cirúrgicas de preservação miocárdica, da melhora do bypass cardiopulmonar, além de melhor suporte pós-operatório.

Dois estudos publicados recentemente por HORWARTH et al. (11), MONTAGUE \& WILSON (12) apresentaram mortalidade operatória de 10,8\% e $12 \%$, respectivamente. A mortalidade operatória verificada em nosso estudo foi similar, $9,1 \%$ em 361 pacientes. 
Iglézias J C R, Oliveira Jr. J L, Dallan L A O, Lourenção Jr. A, Stolf N A G - Preditores de mortalidade hospitalar no paciente idoso portador de doença arterial coronária. Rev Bras Cir Cardiovasc 2001; 16(2): 94-104.

TABELA 11

ANÁLISE MULTIVARIADA (REGRESSÃO LOGÍSTICA), ODDS RATIO (INTERVALO DE 95\% DE CONFIANCAA) DA MORTALIDADE OPERATÓRIA QUANTO: A DIABETE MELITO, DISLIPIDEMIA, ANGINA PECTORIS, CLASSE FUNCIONAL DE ICC, $\mathrm{PD}_{2}$ E URGÊNCIA DA OPERAÇÃO DE 361 PACIENTES IDOSOS REVASCULARIZADOS

\begin{tabular}{lcccc}
\hline VARIÁVEL & CATEGORIA & “ODDS RATIO” & INTERVALO & p \\
\hline Diabete melito & sim/não & 3.285 & $1.504-7.177$ & 0.0029 \\
\hline Dislipidemia & sim/não & 2.103 & $0.964-4.589$ & 0.0619 \\
\hline Angina pectoris & instável/estável & 5.654 & $1.286-24.862$ & 0.0035 \\
NYHA & III-IV/I-II & 3.251 & $1.473-7.176$ & 0.0219 \\
PD $_{2}$ & $>15 /<=15$ & - & & $>0.05$ \\
Operação & não eletiva/eletiva & - & - & $>0.05$ \\
\hline
\end{tabular}

Quanto à morbidade operatória, os resultados verificados em nossa Instituição são bastante satisfatórios, significativamente menores que os observados na literatura (13-17)

A principal complicação pós-operatória encontrada em nossos pacientes foi o baixo débito cardíaco, perfazendo cerca de $1 / 3$ das complicações verificadas, semelhantes aos dados da literatura $(13,14,17)$

No que diz respeito à taxa de infecção detectada para o mediastino, de forma isolada ou em associação, acreditamos que a mesma representa mais um viés criado artificialmente pelos critérios de inclusão do que retrate um valor elevado, em termos absolutos. Foram incluídos no grupo desde pacientes com alteração no hemograma, até aqueles com infecção mediastinal declarada com repercussão para o estado geral.

É interessante notar que presença de baixo débito cardíaco no pós-operatório imediato não mostrou associação significativa com óbito, ao contrário do que é sugerido por alguns autores (18) O emprego de um segundo modelo estatístico que nos permitiu uma análise "passo a passo" da correlação das 5 variáveis pós-operatórias com a mortalidade, evidenciou um ponto interessante: a associação significativa entre baixo débito cardíaco e óbito até a introdução como variável estudo o suporte respiratório prolongado, após o que a associação com a mortalidade operatória se desfazia $(p>0,05)$ e 0 baixo DC deixava de ser um fator preditivo de óbito. Nesta situação, a associação de suporte respiratório prolongado com óbito diminuiu, mas se manteve significativa.

Sugerimos uma possível interpretação biológica para este achado estatístico dado o fato de que a associação de baixo débito débito cardíaco com óbito só se faz na ausência de um suporte ventilatório prolongado; os pacientes que evoluem com baixo DC pós-operatório ao serem extubados mais tardiamente, tenderiam a apresentar menor mortalidade operatória.

É de se esperar que os pacientes com baixo débito cardíaco apresentem um certo grau de congestão pulmonar e, daí, certa tendência à hipoxemia, extremamente prejudicial a um paciente coronariopata com déficit de função ventricular associada, seja por acidose metabólica, seja pelo aumento da FC (alterações esperadas numa situação de hipoxemia) deprimindo o miocárdio e aumentando seu consumo de $\mathrm{O}_{2}$, respectivamente.

Cabe relembrar que $31,7 \%$ dos pacientes apresentaram baixo débito cardíaco no pós-operatório e cerca de 1/ 3 dos óbitos ocorreu por choque cardiogênico.

A idade avançada tem sido vastamente mostrada na literatura como um fator correlacionado com a mortalidade operatória e ao longo do tempo na $\mathrm{RM}^{(12-16,19-23)}$.

Por diversas razões, a idade avançada pode ser considerada como fator de alto risco em procedimentos cirúrgicos de grande porte, especialmente em operações cardíacas.

Os pacientes idosos geralmente apresentam acometimento coronário, mais extenso, com artérias tortuosas, rígidas, lesões calcificadas, freqüentemente com déficit de função ventricular associado(24), além de fatores extracardíacos que também podem ser determinantes do resultado da $\operatorname{RM}(21,25)$

A gravidade e a progressão da doença coronária são multifatoriais, podendo não se refletir na intensidade da ICC, fator apontado amplamente na literatura como um dos principais, ou o principal, fator prognóstico da RM em pacientes idosos $(18,25)$. Neste estudo verificamos uma significativa associação da 
Iglézias J C R, Oliveira Jr. J L, Dallan L A O, Lourenção Jr. A, Stolf N A G - Preditores de mortalidade hospitalar no paciente idoso portador de doença arterial coronária. Rev Bras Cir Cardiovasc 2001; 16(2): 94-104.

classe funcional da ICC pré-operatória, não só com a morbidade $(p=0,0331)$, mas também com a mortalidade operatória $(p=0,0219)$.

Alguns estudos $(26,27)$ demonstram piores resultados em pacientes do sexo feminino. Para STEVEN et al. (28), esses resultados devem-se à maior média de idade e à ICC mais avançada, nas mulheres ao serem encaminhadas aos Serviços de Cirurgia. Outros autores também associam esse resultado com diferenças significativas no status préoperatório dos pacientes do sexo feminino, seja com a maior proporção de angina instável (26) ou com diferenças anatômicas nas artérias coronárias das mulheres (menor distribuição de ramos periféricos, diâmetro médio menor) (28)

Neste estudo o sexo não foi um fator determinante de maior morbidade e mortalidade operatórias $(p>0,05)$, resultado semelhante ao publicado por outros autores ${ }^{(18,25)}$. Ao contrário dos resultados obtidos por GERSH \& FRYE ${ }^{25}$, acometimento de TCE e tabagismo não foram preditores de maior morbi-mortalidade em nosso estudo. Partindo da premissa de que a população brasileira vem apresentando aumento progressivo da expectativa de vida, considerando que a morbi-mortalidade da RM em pacientes idosos é cada vez menor, podemos concluir que a operação representa boa alternativa terapêutica, sobretudo se indicada precocemente, ao portador de doença arterial coronária, mesmo idoso, pois propicia melhora substancial da qualidade de vida e aumento da expectativa, em alguns grupos.

\section{CONCLUSÕES}

O estudo dos fatores pré e pós-operatórios possivelmente associados (prognósticos) com a morbi-mortalidade operatória em 361 pacientes com idade igual ou superior a 70 anos, permitiu concluir:

- A RM pode ser realizada com elevado nível de segurança, com índices aceitáveis de morbidade e mortalidade operatórias, mesmo em pacientes com idade muito avançada.

- A operação pode proporcionar melhora substancial da qualidade de vida nos pacientes desta faixa etária, o que pode ser verificado, não só pela eliminação da angina, como pela redução dos sinais e sintomas de insuficiência cardíaca congestiva.

- A indicação da RM em pacientes com idade muito avançada deve ser um pouco diferente das décadas mais jovens; em pacientes idosos a melhora da qualidade de vida deve ser sempre muito valorizada.

- Ao se retardar a indicação da operação, por conta de preceitos incorretos sobre o nível de segurança e os resultados que se têm obtido com a RM nesta faixa etária, pode se impor ao paciente piores resultados, no que tange à morbi-mortalidade operatória, por conta da evolução da classe funcional de ICC (um dos principais fatores prognósticos) enquanto aguarda possível operação.

Iglézias J C R, Oliveira Jr. J L, Dallan L A O, Lourenção Jr. A, Stolf N A G - Hospital mortality determinants in the elderly patient after coronary surgery. Rev Bras Cir Cardiovasc 2001; 16(2): 94-104.

ABSTRACT: Introduction: The trend in Brazil of erroneously delaying myocardial revascularization in the elderly determined this study. Three hundred consecutive elderly patients (mean age: 73.92, standard deviation: 3.32).

Material and Methods: Between October 1992 and July 1995, 361 consecutive patients underwent isolated coronary artery bypass grafting, of whom $111(30.7 \%)$ were females and 250 (69.35) males. There were 128 (35.5\%) diabetic patients and 128 (36.7\%) were in NYHA III/IV. Univariate analysis perioperatory of 19 factors followed by multivariate logistic regression analysis of the significant variables $(p<0.005)$ were done.

Results: Major complication occurred in 178 (49.3\%) and were independent predictors of operative morbidity: Diabetes mellitus, NYHA functional classification, urgent cases and $\mathrm{DP}_{2}$. There were 33 (9.1 $\%$ ) in hospital deaths, and diabetes mellitus, NYHA functional classification, unstable pre-operative angina and cerebral vascular accident, renal failure, infection and insufficiency respiratory failure were independent predictors of operative mortality.

Conclusion: The coronary artery bypass grafting is possible in elderly patients with a favorable outcome, especially when done in patients with normal to moderately depressed left ventricular function.

DESCRIPTORS: Coronary disease, surgery. Myocardial revascularization, mortality. Hospital mortality. Myocardial revascularization, aged. 
Iglézias J C R, Oliveira Jr. J L, Dallan L A O, Lourenção Jr. A, Stolf N A G - Preditores de mortalidade hospitalar no paciente idoso portador de doença arterial coronária. Rev Bras Cir Cardiovasc 2001; 16(2): 94-104.

\section{REFERÊNCIAS BIBLIOGRÁFICAS}

1 Rabelo R C, Bernardes R C, Reis Filho F A R, Rabelo W, Marino R L - Revascularização do miocárdio no idoso. Arq Bras Cardiol 1993; 61 (Supl. 3): 182.

2 Chaitman B R, Bourassa M G, Davis K et al. Angiographic prevalence of high-risk coronary artery disease in patient subsets (CASS). Circulation 1981; 64: 360-7.

3 Weinstein M C, Coxson P G, Williams L W, Pass T M, Stason W B, Goldman L - Forecasting coronary heart disease incidence, mortality, and cost:: the coronary heart disease policy model. Am J Public Health 1987; 77: $1417-26$

4 Kelly M E, Taylor G J, Moses H W et al. - Comparative cost of myocardial revascularization: percutaneous transluminal angioplasty and coronary artery bypass surgery. J Am Coll Cardiol 1985; 5: 16-20.

5 Califf R M, Harrell Jr. F E, Lee K L et al. - The evolution of medical and surgical therapy for coronary artery disease: a 15-year perspective. JAMA 1989; 261: 2077-86.

6 Hosmer D W \& Lemeshow S - Fundamentals of biostatistics. 2a ed. Boston: PWS Publishers, 1982.

7 Ep. Info 6.0 Andrew G. Dean MD - Epidemiology program office, Mailstop centres for disease control and prevention, Atlanta.

8 SAS Institute Inc. SAS STAT User's guide. Version 6.0, 4. eci. vol. 2. Cary, NC: SAS Institute INC., 1989.

9 Campos-Filho N \& Franco E L - Epidemiologic programs for computers and calculators: a microcomputer program for multiple logistic regression by unconditional and conditional maximum likelihood methods. Am J Epidemiol 1989; 129: 439-44.

10 Gersh B, Freye R L, Kronmal R A et al. - Coronary angiography and coronary artery bypass surgery in elderly patients. Am J Cardiol 1981; 47: 494-503.

11 Horvath K A, DiSesa V J, Peigh P S, Couper G S, Collins Jr. J J, Cohn L H - Favorable results of coronary artery bypass grafting in patients older than 75 years. $J$ Thorac Cardiovasc Surg 1990; 99: 92-6.

12 Montague $3^{\text {rd }} \mathrm{N} \mathrm{T}$, Kouchoukos N T, Wilson T A et al. - Morbidity and mortality of coronary bypass grafting in patients 70 years of age and older. Ann Thorac Surg 1985; 39: 552-7.

13 Tsai T P, Chaux A, Kass R M, Gray R J, Matloff J M Aortocoronary bypass surgery in septuagenarians and octogenarians. J Cardiovasc Surg 1989; 30: 364-8.

14 Rich M W, Sandza J G, Kleiger R E, Connors J P Cardiac operations in patients over 80 years of age. $J$ Thorac Cardiovasc Surg 1985; 90: 56-60.

15 Kuan P, Bernstein S B, Ellestad M H - Coronary artery bypass surgery morbidity. J Am Coll Cardiol 1984; 3: $1391-7$.
16 Acinapura A J, Rose D M, Cunningham Jr. J N, Jacobowitz I J, Kramer M D, Zisbrod Z - Coronary artery bypass in septuagenarians: analysis of mortality and morbidity. Circulation 1988; 78 (3 Pt 2): 1179-84.

17 Tsai T P, Nessim S, Kass R M et al. - Morbidity and mortality after coronary artery bypass in octogenarians. Ann Thorac Surg 1991; 51: 983-6.

18 Gann D, Colin C, Hildner F J, Samet P, Yahr W Z, Greenberg $\mathrm{J} J$ - Coronary artery bypass surgery in patients seventy years of age and older. $J$ Thorac Cardiovasc Surg 1977; 73: 237-41.

19 Cosgrove D M, Loop F D, Lytle B W et al. - Primary myocardial revascularization: trends in surgical mortality. J Thorac Cardiovasc Surg 1984; 88 (5 Pt 1): $673-84$.

20 Gersh B J, Kronmal R A, Schaff H V et al. - Long-term (5 year) results of coronary bypass surgery in patients 65 years old or older: a report from the Coronary Artery Surgery Study. Circulation 1983; 68 (3 Pt 2): II190-9.

21 Edmunds Jr. L H, Stephenson L W, Edie R N, Ratcliffe M B - Open-heart surgery in octogenarians. $N$ Engl J Med 1988; 319: 131-6.

22 Naunheim K S, Dean P A, Fiore C A et al. - Cardiac surgery in the octogenarian. Eur J Cardiothorac Surg 1990; 4: 130-5.

23 Weintraub W S, Jones E L, Craver J, Guyton R, Cohen $C$ - Determinants of prolonged length of hospital stay after coronary bypass surgery. Circulation 1989; 80: 276-84.

24 Rose D M, Gelbfish J, Jacobowitz I J et al. - Analysis of morbidity and mortality in patients 70 years of age and over undergoing isolated coronary artery bypass surgery. Am Heart J 1985; 110: 341-6.

25 Gersh B J, Kronmal R A, Frye R L et al. - Coronary arteriography and coronary artery bypass surgery: morbidity and mortality in patients ages 65 years or older: a report from the Coronary Artery Surgery Study. Circulation 1983; 67: 483-91.

26 Loop F D, Golding L R, MacMillan J P, Cosgrove D M, Lytle B W, Sheldon W C - Coronary artery surgery in women compared with men: analyses of risks and long-term results. J Am Coll Cardiol 1983; 1 (2 Pt 1): $383-90$.

27 Krumholz H M, Douglas P S, Lauer M S, Pasternak R C - Selection of patients for coronary angiography and coronary revascularization early after myocardial infarction: is there evidence for a gender bias? Ann Intern Med 1992; 116: 785-90.

28 Khan S S, Nessim S, Gray R, Gray R, Czer L S, Chaux A, Matloff $\mathrm{J}$ - Increased mortality of women in coronary artery bypass surgery: evidence for referral bias. Ann Intern Med 1990; 112: 561-7. 


\section{Discussão (Transcrições de fita gravada)}

\section{DR. GERALDO MONTEIRO RAMALHO}

Niterói, RJ

Gostaria de agradecer à Comissão Organizadora a indicação do meu nome para comentar o trabalho apresentado. Trata-se de um estudo retrospectivo, não-randomizado, sem grupo controle, levando a limitações na análise dos resultados. Observou-se uma confirmação epidemiológica de que o idoso tem anatomia cirúrgica representada por lesões multiarteriais em $75.9 \%$ dos casos e de tronco coronário esquerdo em $32 \%$. A melhora da classe funcional foi verificada em período curto, 11 dias em média, e não foi o objetivo do trabalho, o que leva a reduzir o valor do achado. A morbidade e a mortalidade pós-operatórias foram maiores nas mulheres, nos diabéticos e nos pacientes submetidos à reoperação de urgência, assim como nos casos de choque e infecção o que corresponde aos achados da literatura e também da nossa experiência. A fração de ejeção foi considerada baixa quando menor que $50 \%$, subestimando a função ventricular. A mortalidade foi de $9,1 \%$, condizente com os dados da literatura. Em nossa recente experiência, em 18 meses, observamos uma mortalidade de $4,6 \%$ em 65 pacientes com idade média de $75,24 \pm 4,5$ anos. A importância do trabalho está mostrar a validade da revascularização cirúrgica em pacientes idosos com bons resultados terapêuticos, o que permite atender uma massa crescente da população de idosos, estimada em $20 \%$ a $25 \%$ da população na primeira metade do século que se inicia. Devemos lembrar que no início da operação de revascularização do miocárdio o limite de idade era de 60 anos e que, atualmente, esse critério foi alongado, sendo apenas considerado o estado clínico do paciente, independente da sua idade cronológica. Concordamos que a proteção miocárdica é de fundamental importância com relação aos resultados obtidos nos pacientes idosos com função ventricular comprometida. Perguntaria ao autor: Que tipo de enxerto empregou? Houve na série operada casos em que não foi utilizada a circulação extracorpórea? Como justifica o expressivo número de enxertos por paciente (2.75pontes/paciente), pois na nossa experiência o número é bastante menor? Parabéns aos autores, principalmente, pela mensagem que o trabalho traz, possibilitando uma melhor qualidade de vida aos pacientes longevos. Muito obrigado.

\section{DR. IGLÉZIAS \\ (Encerrando)}

Agradeço as observações e passarei a responder às questões objetivamente. Com relação ao tipo de enxerto, a artéria torácica interna foi usada todas as vezes que possível mas, o enxerto usado com maior freqüência nesta série foi o enxerto venoso. Em relação à circulação extracorpórea neste momento da observação praticamente não realizávamos a revascularização sem a circulação extracorpórea. Em relação à terceira pergunta, como o objetivo é sempre a revascularização completa daí o número de 2,75 pontes/paciente. 